ESTUDIO DE CASO

\title{
ANÁLISIS DE FACTORES DETERMINANTES DEL PRECIO EN LA CADENA DE VALOR DE LA CARNE BOVINA EN COSTA RICA
}

\author{
Juan Ignacio Herrera-Muñoz', Edgar Solís-Carmona², Guillermo Zúñiga-Arias ${ }^{3}$
}

\section{RESUMEN}

El propósito del presente trabajo fue realizar un análisis de los eslabones en la cadena de valor de la carne bovina y determinar cuáles factores tienen un mayor peso en la formación del precio a través de la misma. El sector industrial es el agente de la cadena que dicta la pauta con respecto al establecimiento del precio interno. Los factores más importantes en la determinación del precio son: el precio internacional, la demanda interna, la intermediación y las políticas sectoriales. A partir de un estudio de caso de una finca con parámetros productivos considerados aceptables, se determinó que un productor de cría bajo las condiciones actuales (2015) de mercado puede obtener un $\mathrm{ROI}$ superior al $16 \%$, con un ingreso de $\$ 72 /$ ha/año (bajo condiciones similares: 100 ha y una carga animal de 1,6 UA/ha). El productor primario tiene muy poca influencia sobre la determinación del precio al que se paga su producto, sin embargo tiene herramientas disponibles para realizar una mejor proyección de producción en función de factores observables que afectan la tendencia del precio local. La elaboración y aplicación de un modelo para la fijación de precios de la carne bovina, parece un objetivo poco probable de llevarse a cabo por la fuerte influencia que tienen algunos sectores en las políticas sectoriales y la poca capacidad de los productores primarios para ejercer presión sobre este asunto.

Palabras clave: Carne vacuna, cadena valor, precio, análisis financiero.

\footnotetext{
'Universidad de Costa Rica. Escuela de Zootecnia. San José, Costa Rica. Autor para correspondencia: juanignacio.herrera@ucr.ac.cr

${ }^{2}$ Universidad Fundepos Alma Máter. San José, Costa Rica.

${ }^{3}$ Universidad Nacional. Escuela de Planificación y Promoción Social. Heredia, Costa Rica.

Recibido: 11 de marzo $2016 \quad$ Aceptado: 6 de julio 2016
} 


\section{ABSTRACT}

The purpose of the present work was to perform an analysis of the links that make up the value chain of bovine meat, and determine which factors have the most influence in the construction of price throughout it. The industrial sector is the agent that sets the standard when it comes to the establishment of the internal price. The most important factors in the determination of price are: international price, internal demand, intermediation and sectoral policies. From a case study of a model farm, with parameters considered acceptable, it was determined that a breeder, under present (2015) market conditions, can obtain a ROI above 16\%, with an average income of $\$ 72 /$ ha/year (under similar conditions: 100 ha and stocking rate of 1,6 AU/ha). Primary producers have little or no influence over the price at which their product is paid; however, they have tools available that can help elaborate better production projections, using observable factors that have an impact on local market tendencies. The elaboration and application of a model for price fixing in bovine meat, appears as a very unlikely objective in the near future, because of the heavy influence some agents have in sectoral policies, whose interests can be negatively affected by this.

Keywords: Bovine meat, value chain, price, financial analysis. 


\section{INTRODUCCIÓN}

La ganadería de carne bovina en Costa Rica es una actividad que viene en claro declive desde hace varios años. Según establecen Holmann et al. (2007), Costa Rica ha experimentado un decrecimiento de la producción de $0,1 \%$ anual durante los últimos 20 años, así como una reducción del hato hasta aproximadamente un $50 \%$ en el mismo período. Por otro lado, la inversión estatal cayó del $5 \%$ del presupuesto nacional a inicios de los 90 , hasta solo $1,5 \%$ en la primera década del siglo XXI. EI mismo comportamiento se evidencia en el crédito agropecuario, cuando en 1990 representaba el $15 \%$ de la colocación total ( $4 \%$ para ganadería), mientras que para la primera década de los años 2000 había caído a 5\% (1,7\% para crédito ganadero). Esta situación provoca que muchos productores se decidan a utilizar la tierra en otras actividades agropecuarias más rentables y por ende se debilita el sector año con año (Grandlgruber et al., 2014). Las tierras destinadas a la cría y engorde son sustituidas por amplias producciones de monocultivos agrícolas (piña, banano, melón, arroz) y el hato bovino de carne se ha venido reduciendo año con año (Madrigal y Fallas, 2012). Las políticas públicas destinadas a incentivar y dinamizar el sector, no parecen hacer mella en esta tendencia. Las causas son varias, sin embargo, se percibe un consenso general que indica que el productor primario medio es poco tecnificado y no utiliza herramientas de gestión que le permitan optimizar su actividad y maximizar su rendimiento, lo que a su vez es atribuido a la ausencia de incentivos de inversión (precio diferenciado por ejemplo) para alcanzar productos de mejor calidad y con valor agregado. Aunado a esto, existen riesgos intrínsecos a la naturaleza de la actividad, al ser los activos seres vivos cuyo desempeño es dependiente en gran parte de factores externos al control del ganadero, además de ser bastante sensible a cambios en el mercado.

La literatura sobre análisis económicos y financieros concernientes a la actividad de ganado de carne en Costa Rica se podría decir es escasa. Un estudio realizado por el Ministerio de Economía, Industria y Comercio (Hidalgo, 2009), cuyo objetivo fue identificar los márgenes de comercialización que obtienen los agentes económicos que participan en la producción, distribución y venta de la carne de ganado vacuno en Costa Rica, encontró que existen diferencias en los márgenes de comercialización entre dichos agentes. Las plantas procesadoras manejan un margen de comercialización bruto promedio de $12 \%$, los intermediarios perciben un margen del 7 $19 \%$ dependiendo si comercializan la carne en canal o en cortes, mientras que los 
detallistas (en los que se consideran carnicerías y supermercados) obtienen de un 11$15 \%$ si compran el producto en cortes y un $23-34 \%$ si lo compran en pie o canal. El autor de este estudio hace hincapié en que la información obtenida es solo una referencia sobre el comportamiento del sector, ya que una determinación precisa de estos indicadores resulta muy compleja debido a la dificultad en la obtención de información (Hidalgo, 2009).

Con respecto a la producción primaria, la información disponible es aún menor y la posibilidad de realizar inferencias a partir de los estudios disponibles es muy baja pues existe gran variedad de sistemas y esquemas de producción, lo que hace que la variabilidad en cuanto a estructuras de costos $\mathrm{y}$, por ende, a utilidades percibidas sea considerable. Un análisis realizado por la Corporación de Fomento Ganadero CORFOGA (2011), que dice utilizar como base un modelo de costos propuesto por el Ing. Javier Gallardo en el 2001, arroja resultados interesantes y un tanto alarmantes. Según dicho análisis, el productor dedicado exclusivamente al engorde de ganado y que vende sus animales a través de una subasta ganadera, percibe un margen de utilidad del $2,67 \%$, mientras que el mismo productor que comercializa su producto a través de la planta de sacrificio obtiene un $7,6 \%$. En el caso del criador, se reporta una pérdida anual cercana al $60 \%$; dato preocupante y que plantea la posibilidad de que se hayan sobreestimado costos, subestimado ingresos o que, de forma menos probable pero no imposible, muchos criadores subsidien la cría de ganado vacuno con otras actividades económicas propias y al no llevar un riguroso control financiero de sus actividades, no sean completamente conscientes de dicha situación.

Se desea con este trabajo, aportar información sobre cuál es el rendimiento real que percibe un productor primario actualmente en relación a los demás actores de la cadena y a partir de esto determinar si existe algún tipo de desbalance en la agregación de valor a través de la misma, así como determinar cuáles son los principales factores y su peso relativo en la fijación del precio. 


\section{MATERIALES Y MÉTODOS}

Se llevó a cabo una revisión bibliográfica de fuentes primarias y secundarias y se realizaron entrevistas a entidades y actores que actúan ya sea directa o indirectamente en la cadena de valor, como productores, asociaciones de productores, instituciones promotoras (CORFOGA, MAG), agentes industriales y comercializadores. A través de este análisis se esclareció cuáles son los eslabones participantes de la cadena, su peso relativo en la misma y cuáles son sus características principales en la actualidad.

Los productores se eligieron bajo los siguientes criterios: que se dediquen exclusivamente a la cría y desarrollo hasta la etapa de destete y que tengan un esquema de producción similar, además que trabajen en una zona geográfica y condiciones similares de ambiente, esto para determinar cuáles diferencias en estos aspectos pueden significar variaciones considerables en las estructuras de costos de dichas explotaciones. Se elige al criador como representante del sector primario de producción, puesto que estudios preliminares y agentes involucrados en la actividad, señalan que es el eslabón de la cadena con un mayor riesgo y que percibe un menor rendimiento. Para efectos del presente estudio se considerará solamente un modelo de comercialización en que participan los siguientes agentes de la cadena: productor primario criador, subasta, planta procesadora y comercio detallista, en las diferentes posibles interacciones que pueden ocurrir entre ellos.

Estudio de caso: A partir de un estudio de caso de una finca de cría modelo, se obtuvo información sobre egresos e ingresos que permitió construir un flujo de efectivo anual, para determinar el rendimiento real que percibe una finca representativa del sector primario. Se consideraron todos los rubros en detalle correspondientes a: mano de obra, alimentos animales, vehículos, insumos para producción de pastos, veterinaria, compra de ganado/semen, equipos y otros. Esto se realizó para contar con información actual y de primera mano que apoye la hipótesis que estipula que, de forma general, el productor que se dedica únicamente a la cría de ganado bovino de carne hasta la fase de destete, es aquel que percibe un menor margen de beneficio, aunado al mayor riesgo que asume, al ser su ciclo productivo considerablemente más largo que el de otros eslabones de la cadena y por tanto, estar más expuesto a las variaciones en el precio a través del tiempo. 
Como indicadores financieros se calcularon:

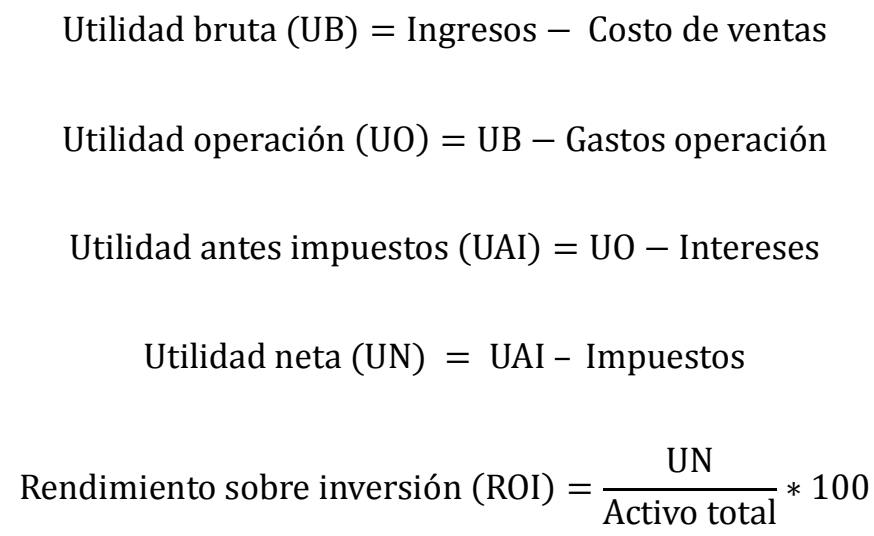

\section{RESULTADOS Y DISCUSIÓN}

\section{Eslabones de la Cadena de Valor}

Producción primaria: En la producción primaria podemos ubicar a todos aquellos agentes responsables de producir animales en pie, cuyo fin último es la obtención de carne para el consumo humano. Según CORFOGA (2013), en el país existen aproximadamente 36.222 fincas dedicadas al ganado bovino de carne, en diferentes sistemas productivos, ya sea criador, engordador, doble propósito y/o ciclo completo. En su gran mayoría las explotaciones se caracterizan por ser pequeñas, al tener no más de 30 animales $(71,1 \%)$ y tener una extensión menor a 30 ha $(70,2 \%)$.

El criador es aquel productor primario que se dedica principalmente a la producción de animales jóvenes a edad de destete (aproximadamente 7-8 meses) y con pesos de entre 180-210 kg (Pérez et al., 2006). Este productor maneja un sistema de producción relativamente complejo, pues debe encargarse de varias fases productivas: un hato reproductor, ya sea compuesto de machos y hembras o solamente hembras (si utiliza inseminación artificial), un hato de animales de reemplazo, que sustituirán eventualmente a los reproductores actuales y del desarrollo de aquellos animales jóvenes al pie de la vaca (en lactancia). El manejo reproductivo generalmente representa costos mayores, pues se deben considerar aspectos como la selección y compra de material genético, manejo nutricional de animales en reproducción, riesgos asociados al proceso biológico de la reproducción; por ejemplo, bajas tasas de 
concepción-natalidad y abortos, así como un mayor requerimiento de mano de obra para el manejo de los animales. Según el último muestreo ganadero realizado por CORFOGA en el año 2012 en el país, de todas las fincas dedicadas a la actividad de carne bovina, solamente un $9 \%$ (3.243) se dedican exclusivamente a la cría.

El engordador es aquel productor primario que se dedica a la producción de animales terminados para la venta y su posterior procesamiento en carne consumible. Se entiende por animal terminado a aquel bovino de carne que alcance un peso vivo aproximado de $550 \mathrm{~kg}$, idealmente a una edad no mayor a 24 meses (MAG, 2007). Éste no mantiene animales reproductores en su finca (vientres o toros), por lo que está exento del manejo reproductivo del hato y los riesgos que éste conlleva. Además de esto, el ciclo productivo tiende a ser considerablemente más corto en la actividad de engorde que en la cría. Si se considera el desarrollo de un vientre y se asume una edad promedio de 30 meses a primer parto, y una edad a destete de 7 meses, se obtendrá su primera cría destetada hasta 37 meses después del nacimiento del vientre. Consecutivamente, y bajo condiciones favorables (con intervalos entre partos de 13 meses), se tendrá una cría destetada de este mismo vientre cada 20 meses. Es decir, se obtendrán los primeros $200 \mathrm{~kg}$ (peso destete) a los 37 meses desde el nacimiento de un vientre y consecutivamente $200 \mathrm{~kg}$ cada 20 meses aproximadamente. Asumiendo un esquema de engorde en el que los animales entran a edad de destete ( 7 meses y $200 \mathrm{~kg}$ ) y salen a un peso de $550 \mathrm{~kg}$ con una ganancia de $0,7 \mathrm{~kg} /$ día como promedio reportada por la literatura (MAG 2007), se tiene que:

$\frac{(550 \mathrm{~kg}-200 \mathrm{~kg})}{0,7 \mathrm{~kg} / \text { día }}=500 \mathrm{~d}, \quad \frac{500 \text { días }}{30,4 \text { días } / \mathrm{mes}}=16,5 \mathrm{~m}$

Es decir, se obtendrá en un período de aproximadamente 16.5 meses, una ganancia total de $350 \mathrm{~kg}$ bajo condiciones favorables. Bajo otros esquemas en el que los animales entran con un mayor peso a la finca de engorde, los ciclos pueden ser aún menores.

El doble propósito hace referencia a que el producto final de estas fincas no se limita a la carne, ya sea de ternero destetado o de animal terminado, sino que comercializan de la misma forma, leche producto del ordeño de las hembras lactantes. Aquellas hembras paridas, en lactancia, dan de mamar al ternero para asegurar su óptimo desarrollo, pero al mismo tiempo se ordeñan para obtener un porcentaje de su producción y venderla ya sea como leche fluida o transformada en algún derivado (queso, natilla, yogurt, etc.). Para que esto sea posible, las hembras deben ser 
capaces de producir una cantidad considerable de leche, por lo que regularmente se utilizan razas o cruces con algún componente lechero.

Una producción de doble propósito puede ser de ciclo completo, si desarrolla los terneros hasta que están terminados; o de cría si vende estos terneros al destete. Según CORFOGA (2013), las fincas dedicadas al doble propósito son las más numerosas, representando un $48 \%$ (17.398) de todas aquellas dedicadas a la producción de carne. Según comentan ganaderos dedicados al doble propósito, se considera que es una de las alternativas más viables económicamente para pequeños productores, puesto que la venta diaria de la leche obtenida, aunque en mucha menor cantidad con respecto a una lechería especializada, les permite tener un flujo de caja más dinámico y constante. Esto puede llegar a tener un impacto administrativo positivo, ya que favorece la disponibilidad de recursos y toma de decisiones en el corto plazo y el manejo operativo de las fincas, que en muchas ocasiones experimentan complicaciones debido a una mala proyección en el uso de los recursos para el caso de las fincas que deben planificar según ciclos productivos completos como el de cría.

Industrialización: El segmento industrial de la cadena de carne bovina se puede dividir claramente en dos tipos de plantas; las plantas urbanas y las plantas rurales. Las plantas urbanas son aquellas que se encuentra dentro de la Gran Área Metropolitana (GAM), principalmente en cantones centrales de las provincias de Heredia y Alajuela. El resto de las plantas procesadoras se encuentran en zonas rurales y en su mayoría procesan una fracción del volumen de aquellas urbanas. Las plantas urbanas son cuatro y se encuentran todas a pocos kilómetros de distancia unas de otras. Según datos de CORFOGA (2015), estas plantas son responsables de un $85-90 \%$ del volumen total de sacrificio de ganado bovino en el país. Es decir, de las aproximadas 380.000 cabezas de ganado que se procesan anualmente en el país en plantas industriales, CoopeMontecillos, Matadero del Valle, El Arreo y GICO (Ganaderos Industriales de Costa Rica) procesan, en conjunto, aproximadamente 330.000 cabezas.

Según el Decreto $N^{\circ} 29588$ del Ministerio de Agricultura y Ganadería y el Ministerio de Salud, en su Capítulo 25: De la clasificación de los mataderos, se establece que existen tres tipos de mataderos: Tipo A, B y C. Aquellos Tipo A están aprobados por el MAG-MS para exportar carnes y sus derivados y cumplen con una series de normativas sanitarias y de requerimientos de infraestructura para garantizar el cumplimiento de dichas normativas. Las cuatro plantas urbanas mencionadas 
anteriormente son Tipo A según estipula el decreto. Las plantas Tipo B son aquellas que tienen un nivel tecnológico intermedio y que, aunque deben cumplir con toda la normativa sanitaria, no cuentan con instalaciones tan completas como aquellas Tipo A; además no están autorizadas para exportar productos cárnicos y sus derivados a otros países. Las plantas Tipo $\mathrm{C}$, son plantas rurales que están autorizadas para operar únicamente en el área que comprende su distrito, además cuentan con apenas las instalaciones básicas que les permitan cumplir con la normativa vigente, pero con un riesgo sanitario mayor. No cuentan con cámaras de enfriamiento, por lo que el flujo de producto dentro de las mismas debe de ser muy rápido y eficiente. La localización de las principales plantas de proceso resulta ser un inconveniente para el productor, ya que en las Regiones Chorotega, Huetar Norte y Huetar Atlántica, donde se concentra la mayor cantidad de explotaciones y cabezas de ganado, no existen plantas de proceso Tipo A, lo que repercute en los costos debido a las largas distancias del transporte desde la finca a las plantas de sacrificio.

Todas las plantas trabajan bajo un sistema que permite dos modalidades: ya sea la a) compra directa de la carne por parte de la planta al propietario del ganado o b) el servicio de sacrificio y deshuese. En la primera modalidad se paga sobre rendimiento, específicamente se pagan los kilogramos que pesa la canal (cuerpo del animal sacrificado, sangrado, desollado, eviscerado, sin cabeza ni extremidades). Generalmente, en bovinos el rendimiento en canal ronda un $55-60 \%$ del peso vivo (Méndez, 2008). Es decir, un animal de $550 \mathrm{~kg}$ con un rendimiento de $55 \%$, tendrá un peso de canal de $302,5 \mathrm{~kg}$. La planta establece un precio de $\mathrm{kg}$ en canal y se paga al productor sobre los $302,5 \mathrm{~kg}$, no sobre los $550 \mathrm{~kg}$ de peso vivo del animal. Es por esto que muchas de las estrategias de producción se basan en maximizar este rendimiento, a través de un adecuado manejo nutricional durante el desarrollo del animal, que permita el mayor desarrollo muscular a la menor edad posible. La otra modalidad bajo la que trabajan las plantas de proceso, es el servicio de procesamiento, también conocido popularmente como "boleto". Bajo este modelo, un productor ingresa el animal a la planta y paga a la misma por el servicio de sacrificio, faenado y (si así lo quisiera) deshuese. Es decir, el ganadero entrega el animal en pie, paga una tarifa establecida y luego retira ya sea la media canal o los cortes deshuesados. Esta tarifa promedia los $\notin 25.000$ colones por cabeza ${ }^{1}$, sin importar su categoría.

Comercialización: Las subastas ganaderas son puntos de encuentro entre aquellos propietarios de ganado que buscan vender sus animales y todos los potenciales

\footnotetext{
${ }^{1}$ Comunicación personal. Departamentos de servicio al cliente: Montecillos, El Arreo, GICO. San José, Costa Rica. Junio 2015. US\$45,06 al tipo de cambio de $\varnothing 554,84$
} 
compradores a través de la cadena de valor. Estos animales que son comprados, regularmente son destinados a alguna de las siguientes actividades: a)traslado a una finca para continuar su desarrollo y eventual venta para carne (engordador), b)traslado directo a planta para faena (intermediarios/carniceros), c)traslado a una finca para su utilización como pie de cría. Esta última opción no es recomendable, ya que los animales vendidos en subasta regularmente no llevan atestados genéticos ni sanitarios, por lo que introducirlo a una finca como pie de cría puede ser un riesgo muy grande. Actualmente en el país están vigentes 23 subastas ganaderas a través de todo el territorio nacional, con excepción de la provincia de Heredia. En algunos cantones como Pococí (3 subastas), San Carlos (2) y Puntarenas (2) se da una concentración de establecimientos, principalmente por una cuestión de demanda y situación geográfica de los cantones (CORFOGA, 2012b). Según un estudio realizado por la Corporación Ganadera en el año 2012 (CORFOGA, 2012b), sobre el comportamiento del segmento de subastas ganaderas, se encontró que un $72,1 \%$ de los animales comprados en subasta son trasladados inicialmente a una finca primaria, un $27,6 \%$ a plantas de cosecha y solamente un $0,3 \%$ son revendidos en otra subasta. Esto evidencia que la mayoría de animales comprados son llevados a otra finca, al menos por un corto tiempo, con el propósito principal de aumentar su peso antes de sacrificarlos, sin embargo, una importante fracción es llevado directamente a sacrificio, lo que indica que los intermediarios y comerciales tienen una considerable participación en las subastas.

Cada supermercado utiliza su propia metodología para calcular el precio base a pagar por la materia prima y el precio a establecer al consumidor. Sin embargo, aunque algunos de ellos tienen un músculo comercial considerable, dentro de las variables que consideran en la ecuación para el cálculo del precio, destaca el precio actual establecido por el que en ese momento sea la planta industrial con mayor volumen de sacrificio, la cual generalmente es El Arreo (CORFOGA, 2015a). De manera general, la relación entre comercio y sector primario, es más bien informal, en donde se crean relaciones entre partes, pero que consideran principalmente las condiciones que debe tener el productor para garantizar de alguna forma cierto nivel de calidad de producto. La práctica de celebrar contratos de futuros entre el sector primario y el resto de la cadena no es común en Costa Rica, por lo que los productores siempre están expuestos a las variaciones en el precio de este bien, hacia abajo o hacia arriba.

Estudio de Caso: La finca objetivo se encuentra ubicada en Santa Rosa de Río Jiménez, en el cantón de Guácimo, provincia de Limón. La zona donde se ubica la Nutrición Animal Tropical 10(2): 1-19 ISSN: 2215-3527/ 2016 
finca se caracteriza por tener un clima muy lluvioso que excede los $4.000 \mathrm{~mm}$ de precipitación anuales. El tipo de vegetación es de bosque húmedo tropical y la temperatura oscila entre $23{ }^{\circ} \mathrm{C}$ a $30{ }^{\circ} \mathrm{C}$. Los suelos característicos de la zona son Latosol (formados bajo el agua) e Hidromórfos (Turbosos, pantanosos, con exceso de humus o pobre de él) (Solano y Villalobos 2008). Actualmente trabaja bajo un sistema de cría extensivo, en el cual los animales se encuentran en pastoreo rotacional en potreros extensos de 3-4 hectáreas, principalmente de pasto "natural" Ratana (Ischaemumindicum). Los animales se venden (principalmente en subasta) cuando alcanzan una edad aproximada de 7 meses de edad y un peso aproximado de 180 220kg. En el Cuadro 1 se observa la distribución del hato, que representa aproximadamente 193 unidades animales (UA), considerando la UA como el equivalente a $450 \mathrm{~kg}$ de peso vivo. Al contar la finca con 120 hectáreas de área efectiva de potreros, se obtiene una carga animal de 1,6 UA/ha, la cual duplica el promedio nacional, que según CORFOGA (2013) se encuentra en 0,79 UA/Ha para la cría.

Cuadro 1. Distribución del hato según fase productiva.

\begin{tabular}{cccc}
\hline \multicolumn{2}{l}{ Distribución del Hato } & \# Animales & \# UA \\
\hline Machos & Adultos & 3 & 4 \\
& Jóvenes & 2 & 2 \\
Hembras & Producción & 104 & 114 \\
& Secas & 15 & 15 \\
Terneros & Lactantes & 30 & 8 \\
& Desarrollo & 18 & 11 \\
Novillas & & 61 & 46 \\
Total & & 233 & 193
\end{tabular}

El hato está compuesto principalmente por una base de animales Cebú (Bos indicus), predominantemente de la raza Brahman, aunque en los últimos años se ha ido ingresando sangre a través de cruzamientos de las razas Charolais y Simmental. Además de la monta natural estacional, se utiliza la tecnología de inseminación artificial (IA). Se presentan índices reproductivos buenos: porcentaje de parición= $81 \%$, intervalo entre partos $(\mathrm{IEP})=450$ días, servicios por concepción= 1,2. 
En la Figura 1 se observa un desglose de los egresos de la finca correspondiente al período fiscal 2014-2015. Se consideraron todos los egresos de los que se obtuvo reporte y se clasificaron en mano de obra, cargas sociales, alimentos animales, vehículos, insumos de pastos, veterinaria, compra de ganado o semen, compra y mantenimiento de equipo y otros. En el caso de los ingresos, sólo se registraron entradas en el rubro de venta de ganado.

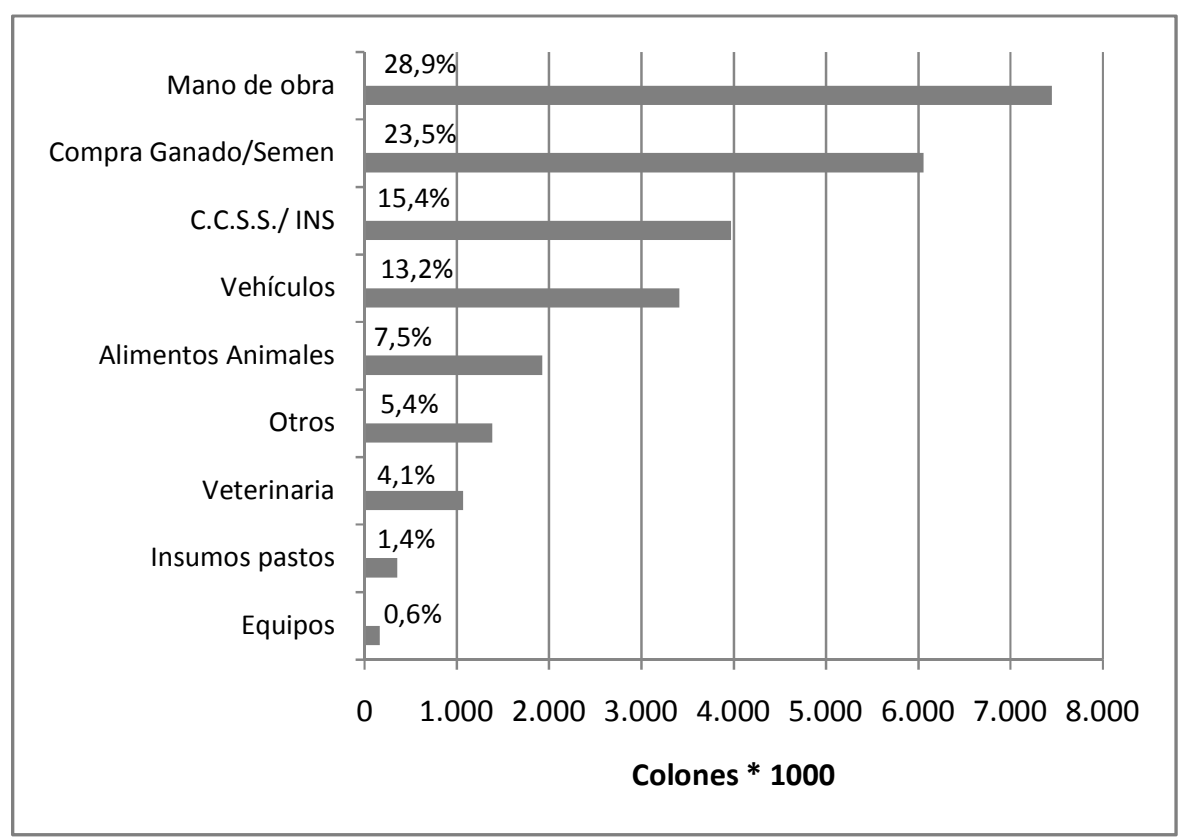

Figura 1. Desglose de los egresos de la finca y su peso relativo al total.

Como se puede apreciar en el Cuadro 2, se obtuvo un margen bruto de $64 \%$ y un margen de operación del 15,3\%, lo que evidencia que los costos asociados a labores operativas representan un porcentaje considerable de los egresos. El margen neto representa un $13,8 \%$, ya que al ser la finca una persona jurídica con un ingreso bruto menor a $ф 52.710 .000$, se le aplica un tasa tributaria del $10 \%$ sobre la renta neta total (Ministerio Hacienda, 2015). En el costo de bienes vendidos se consideraron todos los egresos por alimentos balanceados, mantenimiento de potreros, productos veterinarios, compra de ganado y semen, uso y mantenimiento de equipos y otros. Para el cálculo de los gastos de operación se consideraron los costos de mano de obra y uso de vehículos. 
Cuadro 2. Estado de Resultados

\begin{tabular}{|c|c|c|c|}
\hline Ítem & Colones & & \\
\hline Ingresos por Actividades & $\phi 30.448 .868$ & & \\
\hline - Costo de bienes vendidos & $\phi-10.961 .227$ & & \\
\hline Utilidad Bruta & $\phi 19.487 .641$ & Margen Bruto & $64,0 \%$ \\
\hline - Gastos de operación & $\phi-14.832 .894$ & & \\
\hline Utilidad de Operación & $\phi 4.654 .747$ & M. Operación & $15,3 \%$ \\
\hline Gastos por intereses & $\phi 0$ & & \\
\hline Utilidad Antes de Impuestos & $\phi 4.654 .747$ & & \\
\hline - Gastos por impuestos & $\phi-465.474$ & & \\
\hline Utilidad Neta & $\phi 4.189 .272,30$ & M. Neto & $13,8 \%$ \\
\hline
\end{tabular}

El rendimiento sobre la inversión (utilidad neta/activo total)¹00 arrojó un valor de: $\frac{4.189 .272}{25.794 .121} * 100=16,2 \%$. Si bien es cierto, este valor podría considerarse como aceptable, se debe recordar que en el período sujeto a análisis, se dio una situación de precios al alza.

\section{Factores Determinantes del Precio}

Precio Internacional: Actualmente, 5 plantas industriales cuentan con permisos de exportación hacia otros países (Coopemontecillos, El Arreo, Del Valle, GICO y Cinco Estrellas) otorgado por la Dirección de Inocuidad de Productos de Origen Animal (DIPOA) del Servicio Nacional de Salud Animal (SENASA 2014) y representan más del $85 \%$ del volumen procesado a nivel nacional. Al tener acceso al mercado internacional de productos de origen bovino, el precio en canal que estas plantas pagan a sus proveedores se ve fuertemente afectado por el precio internacional. Si bien es cierto existe un marcado efecto del precio internacional, éste no siempre es inmediato, pues existe evidencia de datos con rezagos de más de 3 meses (inclusive hasta 14 meses) que influyen en el precio local actual (CORFOGA, 2015b). Esto es indicativo de que es probable la existencia de la figura de contratos futuros entre la industria y sus socios comerciales externos. Esta situación hace que el sector industrial exportador tenga una ventaja competitiva por sobre el sector comercial detallista e inclusive sobre el sector industrial no exportador (en cuanto a quién dicta el precio), ya que estos últimos solamente tienen como referencia el precio local actual, que se ve más 
inmediatamente afectado por la demanda interna. Esto tiene un efecto de presión al alza sobre el precio pagado al productor. Cuando los precios internacionales tienden a la baja, la situación cambia un poco, ya que la demanda industrial para exportación puede bajar y si, aunado a esto los precios locales se encuentran relativamente altos, existe un incentivo a la importación, lo que en el corto a mediano plazo empujaría el precio al productor a la baja. Este efecto de los precios internacionales queda en evidencia al verse que solamente un mes después de iniciar una tendencia a la baja en los precios de exportación de Costa Rica, los precios en las plantas que exportan vs aquellas que no exportan, eran en promedio un $8 \%$ inferior (CORFOGA, 2015b).

Demanda Interna: Según Madrigal y Fallas (2012) el hato ganadero costarricense viene con una clara tendencia a la baja durante los últimos años. Mientras en el año 2009 se procesaban alrededor de 94.600 toneladas de carne, para el 2014 se procesaron 88.200 , es decir, una disminución del $1,4 \%$ anual. De la misma forma, pero aún en mayor magnitud, el consumo per cápita (CPC) viene en disminución, pues se contrajo en aproximadamente un $15 \%$ en el espacio de 5 años. Esto evidencia que si bien el incremento en las importaciones $(16,9 \%)$ es mayor al incremento en las exportaciones $(9,6 \%)$ en el mismo período (CORFOGA, 2015a), no viene a cubrir el vacío que deja la producción nacional en el incremento en la demanda por crecimiento demográfico. Esto por cuanto el volumen de las exportaciones es 4 veces mayor al de las importaciones. Esta dinámica regula el CPC interno, ya que al no existir en el mercado suficiente cantidad de producto para cubrir la demanda, se empuja el precio al alza. Las razones de la disminución en la capacidad productiva a nivel país son múltiples y se vienen gestando desde hace algún tiempo, sin embargo, de forma más reciente y puntual, los fenómenos climáticos han golpeado fuertemente al sector. Durante los años 2014-2015 el fenómeno ENOS (Niña y Niño) ha ocasionado un desbalance en el comportamiento normal del clima (IMN 2015) en zonas productoras de ganado bovino de carne, como la región Chorotega, Huetar Norte y Huetar Atlántica, ya sea a través de la sequía prolongada o el exceso de lluvias. Siendo que la producción bovina en Costa Rica se da en su amplia mayoría en sistemas extensivos bajo pastoreo, se vio gravemente afectada su eficiencia productiva por esta situación; se dio una considerable disminución en la oferta. En la Figura 2 se puede apreciar la tendencia al alza durante el 2015 del precio y su eventual estabilización, así como la estrecha relación entre el precio de subasta y el pagado por la industria. 


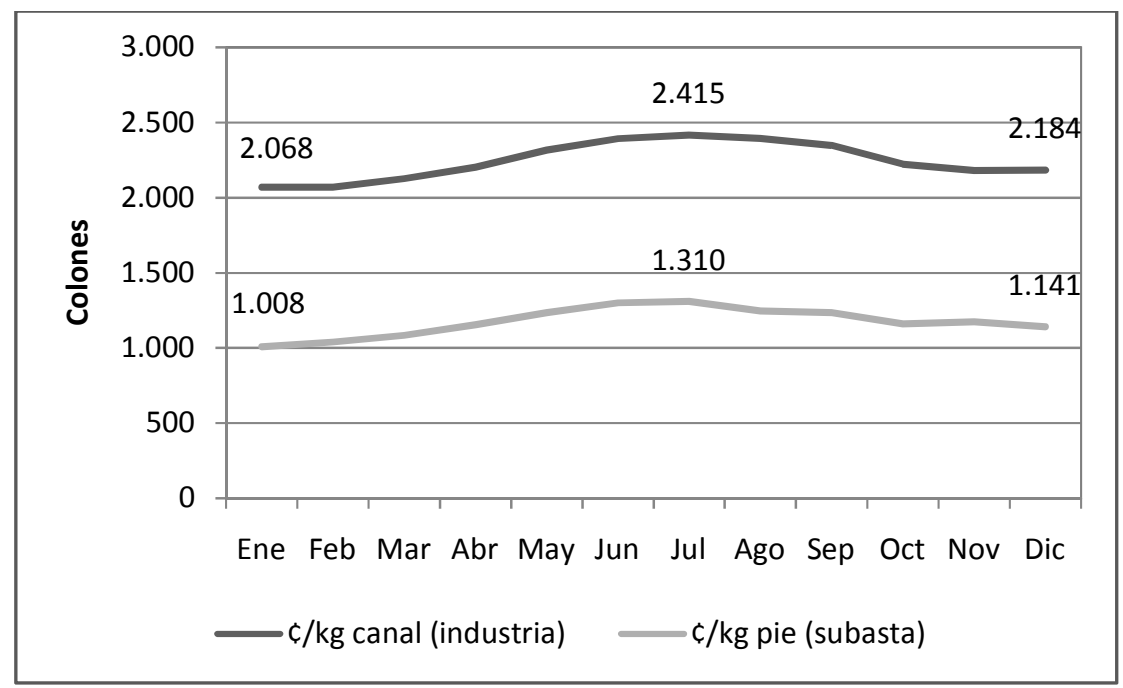

Figura 2. Comportamiento del precio en industria y subasta durante el 2015. Adaptado de datos de CORFOGA.

Intermediación: En toda cadena de valor, el precio no solamente se determina por el balance costo producción-oferta-demanda. En muchas ocasiones, existen agentes que al incrementar la intermediación en la comercialización ya sea de materias primas o producto final, introducen un incremento al precio. En la cadena de valor de la carne bovina, este efecto se da mayormente a nivel de comercialización en la subasta, en donde muchas veces personas o grupos se dedican exclusivamente a la compra de animales en pie en la subasta para trasladarlos ya sea directamente a la planta de sacrificio o venderlos a un tercero que finalice su desarrollo (siempre con un sobreprecio). Es decir, obtiene un margen de ganancia solamente por transportar a los animales e, indirectamente, empujan el precio al alza. Es cierto que estos agentes incurren en gastos financieros, pues pagan de contado y pueden obtener el pago hasta más de una semana después, sin embargo, al comparar con los tiempos de producción y riesgos asociados del productor primario, estos gastos se podrían considerar insignificantes. Esto ocurre debido a la estructura del sector industrial, que se encuentra muy concentrado en zonas urbanas y obliga al micro y pequeño productor que saca muy pocos animales, pero que compone más del $70 \%$ de las fincas (CORFOGA, 2013), a llevar sus animales a la subasta por el elevado costo de transporte hasta los centros de sacrificio. 
Políticas Sectoriales: En el año 1995 se dejó de utilizar un modelo de fijación de precios desarrollado por el MEIC (Ministerio de Economía, Industria y Comercio) para la carne bovina ${ }^{2}$. Este modelo tenía un fundamento técnico y en su cálculo se consideraban una serie de factores fijos y variables. Desde entonces, el precio es definido por el mercado, sus actores e interacciones. La Corporación de Fomento Ganadero (CORFOGA) fue creada en el año 1998, mediante la Ley 7837 . El artículo 20 de dicha ley establece: "Para los efectos de distribuir en forma equitativa los ingresos de la venta de carne y los subproductos de la res entre los ganaderos e industriales, deberá definirse un mecanismo de liquidación que garantice dicho objetivo $y$, una vez establecido y debidamente publicitado, se aplicará de manera obligatoria". Actualmente la Comisión de Liquidación, encargada de ejecutar lo establecido en dicho artículo, no ha logrado el objetivo ${ }^{3}$. Se han realizado esfuerzos como el "Proyecto de Clasificación de Canales", que de alguna forma venía a plantear las bases para el establecimiento de un esquema de pago diferenciado por calidad, sin embargo luego de aproximadamente 11 años de trabajo, el proyecto fue cerrado por la dirección de la Corporación Ganadera. La comisión está formada por tres representantes del sector industrial, tres representantes del sector ganadero y por el Viceministro de Agricultura y Ganadería. Sin embargo, son muchas las voces que sugieren que el sector industrial tiene una importante influencia en la toma de decisiones y establecimiento de políticas, razón principal por la que el modelo de liquidación no ha visto la luz aún. Recientemente el Centro de Investigación en Economía Agrícola y Desarrollo Empresarial de la Universidad de Costa Rica, realizó un análisis sobre la viabilidad técnica del establecimiento de un sistema de liquidación de precios de la carne bovina (Paniagua et al., 2016). El análisis concluye que, bajo las condiciones actuales, es técnicamente inviable el establecimiento de un sistema de liquidación de precios por varias razones, pero principalmente debido a que CORFOGA no tiene las herramientas legales para exigir la información que permitiría definir los márgenes de comercialización de los agentes participantes de la cadena (principalmente industriales y comerciales) y por tanto, determinar con certeza si en efecto existe un desbalance de fuerzas dentro de la misma.

\footnotetext{
${ }^{2}$ Soto, C. Comunicación personal. Industrias Cárnicas Integradas (ICI) de Centroamérica. Alajuela, Costa Rica. Octubre 2015.

${ }^{3}$ Fallas, M. 2015. Comunicación Personal. Jefe del Departamento de Proyectos. CORFOGA. Febrero 2015.

Nutrición Animal Tropical 10(2): 1-19 ISSN: 2215-3527/ 2016
} 


\section{CONSIDERACIONES FINALES}

La cadena de valor de la carne bovina es compleja y si bien la relación entre sus eslabones está bien definida, obtener información es complicado, pues los sectores comercial e industrial son muy reservados. El precio a través de la cadena se establece principalmente a partir de situaciones de mercado (tanto interno como externo) y no a través de análisis de costos. El sector industrial pareciera ser el agente de la cadena que dicta la pauta con respecto al establecimiento del precio interno, principalmente por el alto volumen de proceso concentrado en pocos establecimientos y porque tiene acceso a la "válvula de escape" que representa la exportación a otras latitudes, considerando el peso que tiene el precio internacional sobre la determinación del precio local. Sin embargo, algunas investigaciones previas señalan que los agentes comerciales finales son aquellos con un mayor margen de ganancia. El productor primario tiene muy poca influencia sobre la determinación del precio al que se paga su producto, sin embargo, tiene herramientas disponibles para de alguna forma mejorar la proyección de la producción en función de factores observables que afectan la tendencia del precio local, como los precios internacionales y el volumen de sacrificio de las plantas industriales (disponible a través de la Corporación Ganadera). Es fundamental que el productor primario tome acción para mejorar sus parámetros reproductivos y productivos, en ese orden de prioridad, además de tomar control sobre sus costos, procurando ser más eficiente año con año. La elaboración y aplicación de un modelo para la fijación de precios de la carne bovina, parece un objetivo poco probable de llevarse a cabo por la fuerte influencia que tienen algunos agentes en las políticas sectoriales, aunado a la falta de herramientas disponibles actualmente para que la Corporación Ganadera pueda llevar esto a cabo.

\section{AGRADECIMIENTOS}

Los autores agradecen al señor Alejandro Rodríguez, propietario de la ejemplar finca "Ganadera don Reca", por su cooperación desinteresada en la obtención de datos fundamentales y al M.Sc. Alejandro Saborío por sus valiosas observaciones para la mejora del presente análisis. 


\section{LITERATURA CITADA}

CORFOGA. 2011. Costos de Producción y Rentabilidad de Fincas Ganaderas.

Revisado en URL: http://corfoga.org/proyectos-y-publicaciones-2/modelo-decostos/ El 15 de noviembre del 2014.

CORFOGA. 2012a. Situación Actual y Perspectivas en la Ganadería. Boletín de Mercado, Departamento de Inteligencia de Mercados.

CORFOGA. 2012b. Estudio de Subastas Ganaderas en Costa Rica. Informe: Cadena de Estudios de Comercialización.

CORFOGA. 2013. Informe sobre Muestreo Ganadero 2012. Boletín. Departamento de Inteligencia de Mercados.

CORFOGA. 2015a. Informe Estadístico Octubre: Procesamiento y precios. Informe. Departamento de Investigación y Divulgación.

CORFOGA. 2015b. Boletín de Precios de Ganadería y Carne Bovina. Departamento de Investigación y Divulgación. Boletín, Octubre, 2015.

GRANDLGRUBER B., GARCÍA H., NAZIF I. 2014. Economía institucional de la formación de precios agrícolas: Propuesta Metodológica. CEPAL, Sede Subregional, México.

HIDALGO J. 2009. Márgenes de comercialización de la carne bovina en Costa Rica. Informe del Ministerio de Economía, Industria y Comercio (MEIC).

hOLMANN F., RIVAS L., PÉREZ E., CASTRO C., SCHUETZ P., RODRÍGUEZ J. 2007. La cadena de carne bovina en Costa Rica: Identificación de temas críticos para impulsar su modernización, eficiencia y competitividad. ILRI, CIAT, CORFOGA. 
IMN (Instituto Meteorológico Nacional). 2015. Boletín ENOS. Revisado en URL:http://www.imn.ac.cr/43 El 15 de agosto de 2015.

MADRIGAL J. \& FALLAS M.A. 2012. Informe Encuesta Ganadera 2012. Informe. Corporación de Fomento Ganadero.

MAG (Ministerio de Agricultura y Ganadería). 2007. Plan Estratégico para el desarrollo de la agro cadena de la ganadería bovina de carne en la Región Chorotega.FCGG y MAG, 72p.

MÉNDEZ J. 2008. Agro-cadena de la ganadería bovina de carne de la Región Chorotega. Boletín Extensión Agropecuaria. Ministerio de Agricultura y Ganadería de Costa Rica.

MINISTERIO DE HACIENDA. 2015. Régimen Tributario Tradicional. Dirección de Servicio al Contribuyente. Revisado en http://www.hacienda.go.cr/contenido/12994-regimen-tradicional. $\quad$ El 20 de setiembre de 2015.

PANIAGUA J., RODRÍGUEZ C., VILLALOBOS V. 2016. Viabilidad Técnica del Artículo 20 de la Ley 7837, Basado en el Establecimiento de un Sistema de Liquidación de Precios de Carne. CIEDA, Universidad de Costa Rica.

PÉREZ E., hOLMANN F., SCHUETZ P., FAJARDO E. 2006. Evolución de la Ganadería Bovina en Países de América Central: Costa Rica, Guatemala, Honduras, Nicaragua. CIAT (Centro Internacional de Agricultura Tropical).

SENASA. 2014. Establecimientos aprobados para exportar productos y subproductos de Origen Animal. Reglamento DIPOA-PG-001-RE-007. San José, Costa Rica.

SOLANO J., VILLALOBOS R. 2008. Regiones y subregiones climáticas de Costa Rica. Instituto Meteorológico Nacional, San José, Costa Rica. Revisado en http://www.imn.ac.cr/publicaciones/estudios/Reg_climaCR.pdfhttp://www.imn.ac.cr /publicaciones/estudios/Reg climaCR.pdf el 4 de setiembre del 2015. 